\title{
Analyze Portrayal of Stable Election Protocol for Wireless Sensor Network using Matlab
}

\author{
Aditi Soni \\ M. Tech Scholar \\ PIET \\ Samalkha
}

\author{
Arun Rana \\ Assistant Professor \\ PIET \\ Samalkha
}

\begin{abstract}
Wireless Sensor Networks (WSNs) are made out of heaps of sensor nodes, with restricted energy, that cooperates to perform a sensing project. In Wireless Sensor Network, the energy efficiency is the important thing difficulty for designing the protocol because sensor nodes have one-time battery backup. There are many cutting-edge protocols which increase the life of the wireless sensor Network through correctly the use of battery energy of the sensor node. In this research work, a brand new strategy and protocol based totally on Stable Election Protocol (SEP) in wireless Sensor network have been proposed. For proposed method, we've assumed heterogeneous environment i.e. the impact of heterogeneity of nodes, in terms in their strength, in wireless sensor networks which might be hierarchically clustered. In those networks, some of the nodes grow to be cluster heads, combination the records of their cluster members and transmit it to the sink. We count on that a percentage of the population of sensor nodes is ready with extra strength assets. We additionally anticipate that the sensors are randomly distributed and aren't cellular, the coordinates of the sink and the size of the sensor discipline are known. The uniqueness of the proposed method is that cluster head is chosen amongst normal Nodes, Intermediate Nodes and Advanced Nodes based upon their common strength. This assets will increase the number of cluster heads in keeping with spherical and number of packets per spherical. First of all the nodes had been labeled as everyday Nodes, Intermediate Nodes and advanced Nodes. Then, all three kinds of nodes are similarly categorized as Alive nodes and dead nodes. The cluster head is selected among all 3 types of nodes on the premise of average energy. Cluster head collects statistics from member nodes, mixture it and transmit it to the base station. Cluster head choice is maximum critical. Once the cluster head is selected then the cluster head broadcasts a commercial message to the nodes. The nodes get hold of the message and decide to which cluster head it is going to be long for the cutting-edge round. A Modified-SEP (proposed approach) has been applied and in comparison with existing SEP. Three parameters i.e. the number of dead nodes, the number of alive nodes and packets transmitted to base station and so forth has been taken as performance parameters. The simulation end result shows that overall performance and throughput of our proposed protocol deliver the effective and widespread power efficiency in addition to more Network lifetime as compared to different protocols. MATLAB R2013a has been taken as an implementation platform.
\end{abstract}

\section{General Terms}

In this author used Matlab R2013a for implementation and recognition.

\section{Keywords}

Wireless Sensor Network, network lifetime, LEACH, SEP etc.

\section{INTRODUCTION}

Wireless Sensor Networks [8] are systems of tiny, battery [3] [4] controlled sensor nodes [5][6]with restricted on-board preparing, stockpiling and radio abilities. Node sense [2] and send their reports toward a preparing focus which is called "sink." The outline of conventions and applications for such systems must be vitality mindful with a specific end goal to draw out the lifetime of the system, on the grounds that the substitution of the installed batteries is an extremely troublesome process once these hubs have been sent.

In Wireless Sensor Network [9], the energy efficiency [7] [8] is the key issue for planning the convention since sensor hubs have one time battery reinforcement. There are numerous cutting edge conventions which expand the lifetime [6] of the remote sensor arrange by productively utilizing battery energy of the sensor node [2].

\subsection{SEP (Stable Election Protocol)}

SEP convention is a change and improvement of LEACH [2] convention which utilizes bunching construct directing procedure situated in light of the hub heterogeneity of the sensor hub in the systems. In this convention and procedure, a portion of the sensor hubs have high vitality they are alluded to as the propelled hubs and the likelihood of the propelled hubs to end up CHs is more when contrasted with the typical hubs and the ordinary hubs have bring down vitality when contrasted with the propelled hubs in the system. SEP methodology utilizes a disseminated strategy to choose a $\mathrm{CH}$ in WSNs. It is heterogeneity-mindful convention [1] and $\mathrm{CH}$ determination probabilities of hubs are weighted by beginning vitality of every hub contrasted with alternate hubs in WSN. So fundamentally, SEP convention depends on two levels of hub heterogeneity as ordinary hubs and propelled hubs.

\subsection{Favorable position of SEP}

Any ID or worldwide information of vitality of sensor hub is not required in SEP [7] strategy at every choice round of bunch head.

\subsection{Constraints of SEP}

The Cluster head $(\mathrm{CH})$ determination among sensor hubs are not dynamic, which comes about that hubs that are far from the capable hubs will bite the dust first.

\section{PROPOSED METHODOLOGY}

\subsection{Parameters Used}

In this examination work, another system and convention in light of Stable Election Protocol (SEP) in Wireless Sensor Network have been proposed. For proposed framework, we have expected heterogeneous of condition i.e. the effect of heterogeneity of hubs, regarding their vitality, in remote sensor organizes that are progressively grouped. In these systems a portion of the hubs progress toward becoming 
group heads, total the information of their bunch individuals and transmit it to the sink. We expect that a level of the number of inhabitants in sensor hubs is furnished with extra vitality assets. We additionally accept that the sensors are haphazardly (consistently) dispersed and are not portable, the directions of the sink and the measurements of the sensor field are known. Right off the bat, every one of the hubs has been ordered as Normal Nodes and Advanced Nodes. Than Advanced hubs are further categoried as Alive Advanced hubs and Dead Advanced Node. Group head is chosen among cutting edge hubs as it were. Bunch head gather information from part hubs, total it and transmit it to base station. Group head choice is generally critical. Once the group head is chosen then the bunch head communicates a promotion message to the hubs. The hubs get the message and choose to which group head it will have a place for the current round.

We have recreated the proposed convention in a field with measurements $100 \mathrm{~m} \times 100 \mathrm{~m}$ and 100 hubs are conveyed in particular zones as for their vitality. A portion of alternate parameters are:

\begin{tabular}{|c|c|}
\hline Parameters of WSN & Values \\
\hline Number of rounds & 500 \\
\hline Alpha $(\alpha)$ & 1 \\
\hline Initial energy (Eo) & $1.5 \mathrm{~J}$ \\
\hline Initial energy of advance nodes & $\operatorname{Eo}(1+\alpha)$ \\
\hline $\begin{array}{l}\text { Energy for data aggregation } \\
\text { (EDA) }\end{array}$ & $5 \mathrm{~nJ} / \mathrm{bit} /$ signal \\
\hline Number of nodes & 100 \\
\hline $\begin{array}{l}\text { Transmitting and receiving energy } \\
\text { (Eelec) }\end{array}$ & $5 \mathrm{~nJ} / \mathrm{bit}$ \\
\hline $\begin{array}{l}\text { Amplification energy for short } \\
\text { distance (Efs) }\end{array}$ & $10 \mathrm{Pj} / \mathrm{bit} / \mathrm{m} 2$ \\
\hline $\begin{array}{l}\text { Amplification energy for long } \\
\text { distance (Eamp) }\end{array}$ & $0.013 \mathrm{pJ} / \mathrm{bit} / \mathrm{m} 4$ \\
\hline Probability (Popt) & 0.1 \\
\hline Filed dimension & $100 \times 100 \mathrm{sq} \mathrm{m}$ \\
\hline
\end{tabular}

\subsection{Practical Application Of MATLAB}

MATLAB R2013 has been utilized as an execution stage. In this work, leaving SEP and Advanced SEP (proposed) has been executed and looked at through their execution parameters. These parameters are:

1. Number of Dead nodes in accordance with increasing number of rounds.

2. Number of alive nodes in accordance with increasing number of rounds.

3. Number of Packets transferred to base station in accordance with increasing number of rounds.

Here, all the execution steps :

In-statement of a few parameters for setting up the system

- $\quad$ Field dimensions $x$ and y maximum (in meters)

- $\mathrm{X}$ and y coordinates of the sink, number of nodes in the field
- Optimal election probability of alpha node to become cluster head

- $\quad$ Energy model (all values in joules)

- Initial energy

- Energy dissipated per bit to run transmitter and receiver circuit,

- Amplification energy for short distance (efs)

- Amplification energy (emp)

- Data aggregation energy

- M-fraction of the total nodes or values for heterogeneity percentage of nodes than are advanced

- Alpha-times advance nodes have energy greater than normal nodes

- Maximum number of rounds

Creation of the random Sensor Network according to the dimensions of field and number of nodes

- Declaration of a loop according to number of nodes

- $\mathrm{G}$ is the set of nodes which have not been cluster heads

- Initially there are no cluster heads only nodes

- Random Election of Normal Nodes

- $\quad$ Normal nodes have energy category 0

- Random Election of Advanced Nodes

- Alpha-times advance nodes have energy greater than normal nodes

- Advanced nodes have energy category 1

Initialization of First Iteration

1. Declaration of counter for Cluster Heads

2. Declaration of counter for Cluster Heads per round

3. Declaration of counter for number of clusters

4. Assignment of total alive nodes

5. Declaration of counter for packets or data to base station and cluster head.

\subsection{Implimentation of SEP}

- Declaration of a loop according to number of rounds.

- Calculation of Election Probability for Normal Nodes

- Calculation of Election Probability for Advanced Nodes

- Assignment of nodes which have not been cluster heads or operation of heterogeneous epochs

- Operations for sub-epochs

- Declaration of counter of Number of dead nodes

- Declaration of counter of Number of dead Advanced Nodes

- Declaration of counter of Number of dead Normal Nodes

- Declaration of a loop according to number of nodes

- Checking if there is a dead node

- Updation of dead node counter

- Checking if node is advanced

- Checking if node is normal

- Checking if there is an alive node

- Assignment of total dead nodes and alive nodes for each round to new variable

- Checking of first node dead or alive

- Declaration of a loop according to number of nodes

- Checking if there is an alive node

- Checking if the node cluster head or not 
- Election of Cluster Heads for normal nodes

- Updation of cluster head counter

- Updation of counter of packets to the base stations

- Assigning of particular node as cluster head.

- Assigning of selected node dimensions to other variable

- Calculation of average distance between a cluster member and its cluster head

- Assignment of calculated distance to new variable

- Updation of cluster counter

- Calculation of Energy dissipated where Eelec $=($ ETX + EDA $)$ is the energy dissipated per bit to run the transmitter or the receiver circuit.

- Checking if average distance between a cluster member and its cluster head is greater than initial distance

- Checking if average distance between a cluster member and its cluster head is lesser than initial distance

- Election of Cluster Heads for Advanced nodes

- Updation of cluster head counter

- Updation of counter of packets to the base stations

- Assigning of particular node as cluster head

- Assigning of selected node dimensions to other variable

- Calculation of average distance between a cluster member and its cluster head

- Assignment of calculated distance to new variable

- Calculation of Energy dissipated where Eelec $=(E T X+E D A)$ is the energy dissipated per bit to run the transmitter or the receiver

- Checking if average distance between a cluster member and its cluster head is greater than initial distance

- Checking if average distance between a cluster member and its cluster head is lesser than initial distance

- Updation of counter of packets to the base station

- Assignment of updated variable to new variable

- Election of Associated Cluster Head for Normal Nodes

- Declaration of a loop according to number of nodes

- Checking if there is any normal and alive node

- Checking if number of cluster more than one

- Calculation of average distance between a cluster member and alive node

- Initialization of loop according to total number of clusters

- Calculation of average distance between a cluster head and alive node and comparison with average distance between a cluster member and alive node

- Calculation of Energy dissipated where ETX is the energy dissipated per bit to run the transmitter or the receiver circuit or Energy dissipated by associated Cluster Head

- $\quad$ Again checking if associated Cluster head distance is greater than initial distance

- Again checking if associated Cluster head distance is greater than initial distance

- Calculation of Energy dissipated

- Checking if minimum distance is positive

- Calculation of energy dissipated by cluster head with minimum distance

- Updation of counter cluster head per round

1. Display of alive hubs as per number of round.
2. Dispaly of dead hubs as per number of rounds.

3. Display of number of bundle transmitted to base station as per number of rounds.

\subsection{Experimental Results}

An effective protocol for Wireless sensor organize has been composed and completed the utilization of Modified Stable Election Protocol for transmission of bundles to the base station through a proficient bunch head. The uniqueness of the proposed strategy is that group head is chosen among ordinary Nodes, Intermediate Nodes and Advanced Nodes in view of their normal quality. This having a place builds the quantity of bunch heads with regards to round and number of bundles steady with the round. Right off the bat, every one of the hubs had been named as Normal Nodes, Intermediate Nodes, and Advanced Nodes. At that point, each of the 3 sorts of hubs are comparably classified as Alive hubs and dead Nodes. The group head is picked among each of the 3 types of hubs on the premise of normal vitality. Group head gathers bundles from part hubs, a blend it and transmits it to the base station. Bunch head decision is greatest imperative. When the bunch head is picked then the group head advertises a business message to the hubs. The hubs get the message and choose to which group head it will probably be yearn for the advanced round. This portion is known as group development stage. On the premise of got flag vitality, hubs react to bunch take and kill to be an individual from the group head. Bunch head at that point allots a TDMA time table for the hubs for the term of which hubs can send measurements to the group head. After the arrangement of the bunch, every hub certainties and sends it to the group head in the availability apportioned with the guide of the bunch make a beeline for the hub. While records are gotten from hubs, Cluster head at that point totals this insights and ship it to the base station this stage is known as transmission portion.

We have executed the technique and got a few previews of MATLAB charge window and figure window alongside some yield parameters i.e. Number of parcels sent to base station at each round, Number of bundles sent to bunch head at each round, Number of Cluster Head at each round, Normal Nodes, Intermediate Nodes and Advanced Nodes finally round, number of alive hubs at each round and normal vitality of alive hubs at each round.

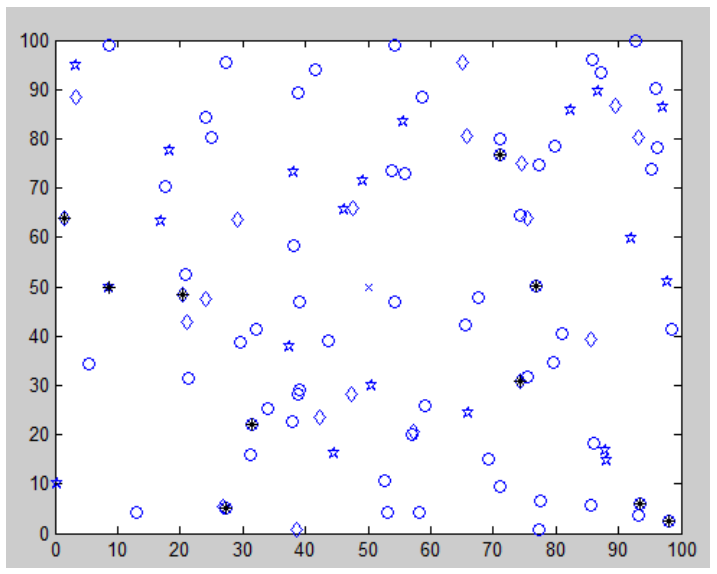

Figure 1. Snapshot of field having size $100 \times 100$ meters having normal nodes $(0)$, intermediate nodes $(*)$ and advanced nodes $(\triangleleft)$ with cluster head $(*)$ 


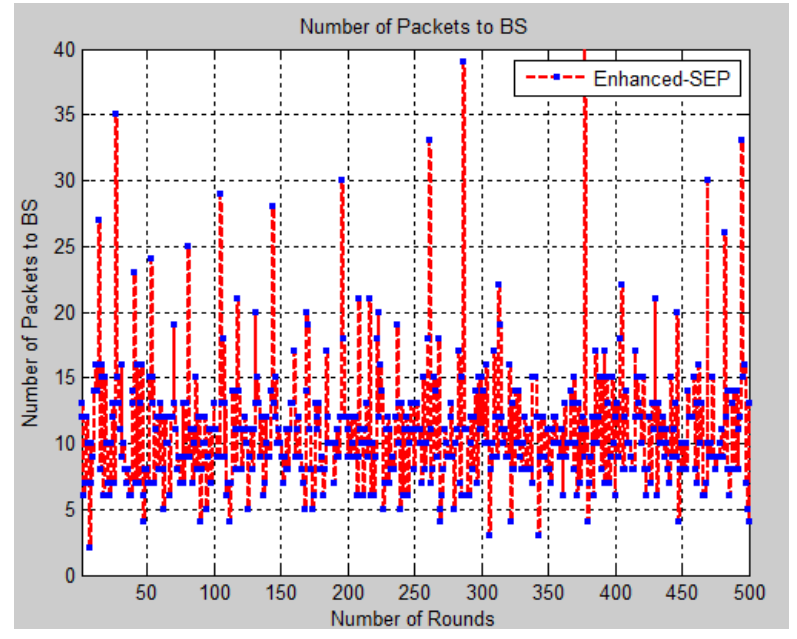

Figure 2. Snapshot of Number of packets sent to base station at each round

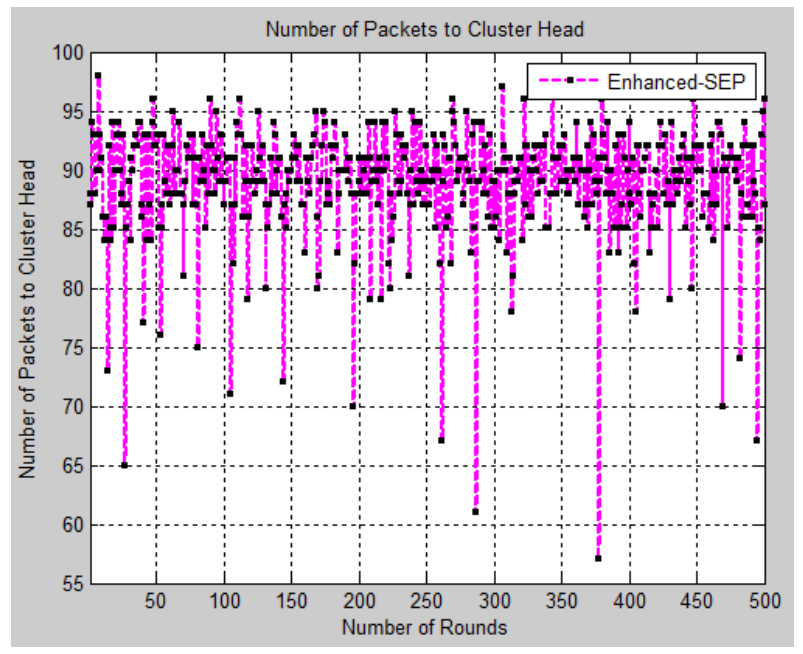

Figure 3. Snapshot of Number of packets sent to cluster head at each round

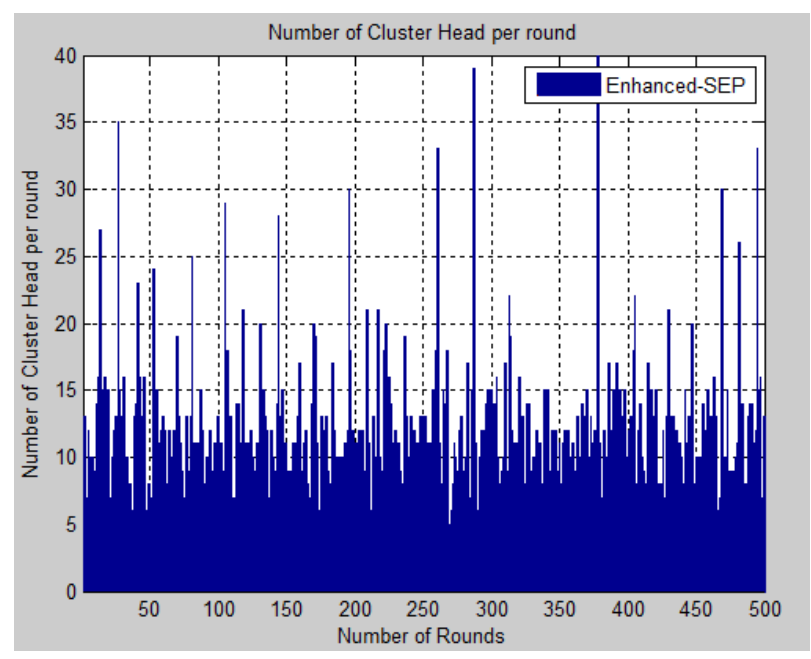

Figure 4. Snapshot of Number of Cluster Head at each round

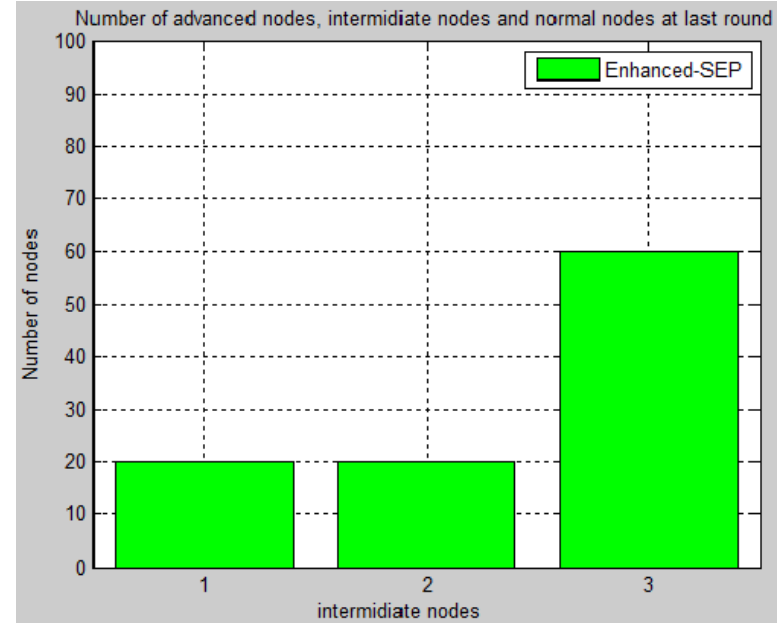

Figure 5. Snapshot of comparison of number of Normal Nodes, Intermidiate Nodes and Advanced Nodes at last round

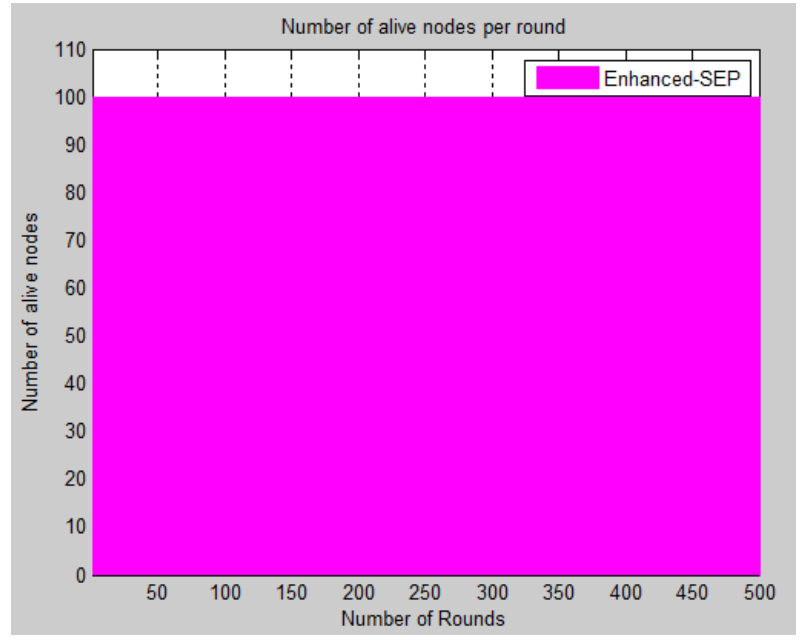

Figure 6. Snapshot of number of alive nodes at each round

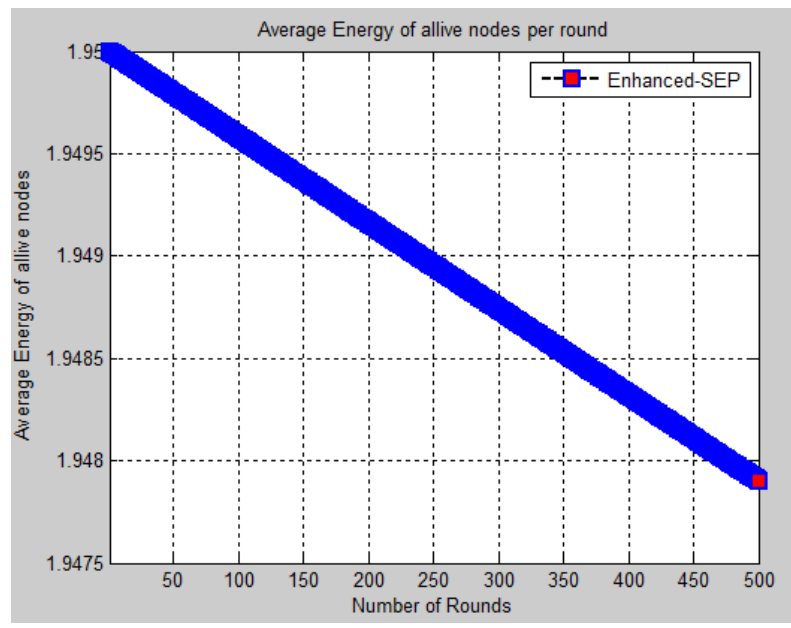

Figure 7. Snapshot of average energy of alive nodes at each round 


\section{CONCLUSION AND FUTURE SCOPE}

The Modified Stable Election based thoroughly directing Protocol for WSNs has been proposed, tried and contrasted and show SEP steering conventions, in this Research work. In this work, we've proposed Modified-SEP for a heterogeneous situation. The distinction of the proposed strategy is that group head is picked among Normal Nodes, Intermediate Nodes and Advanced Nodes in view of their normal vitality. This property expands the quantity of bunch heads per round and number of parcels per round. The field is partitioned into 3 styles of hubs i.e. typical Nodes, Intermediate Nodes and propelled Nodes. Every one of the three sorts of hubs utilize bunching way to deal with transmit parcels to the construct station fundamentally situated in light of their normal vitality. Reproduction outcomes demonstrate that the proposed Modified Stable Election fundamentally based steering Protocol indicates higher general execution as far as quality sparing, alive hubs, dead hubs and bundle transmission. The evidence of above explanations is the charges of parcels transmitted to base stations finally round in each the occurrences. The throughput of changed SEP is additionally raised contrasted and present SEP.

In any case, Modified-SEP isn't generally appropriate in which visit measurements are gotten from the Wi-Fi sensor organize. Our future heading might be to beat this impediment on this convention. At long last, in future, the thought and execution of the versatile base station can be acquainted in proposed device with complete the ensuing level of the innovation of remote sensor group.

\section{REFERENCES}

[1] Jeevan L J Pinto and Manjaiah D. H "Modified Enhanced Stable Election Based Routing Protocol for Wireless Sensor Networks" International Journal of Innovative Research in Computer and Communication Engineering, Vol.2, Special Issue 5, October 2014. Pp. 54-59.

[2] Mishra, Yogesh, Ashish Singhadia, and Rashmi Pandey. "Energy Level Based Stable Election Protocol in Wireless Sensor Network." International Journal of
Engineering Trends and Technology (IJETT)-Volume 17 (2014): 32-38.

[3] Smaragdakis, Georgios, Ibrahim Matta, and Azer Bestavros. SEP: A stable election protocol for clustered heterogeneous wireless sensor networks. Boston University Computer Science Department, 2004.

[4] Singh, Debabrata, and Sanjeet Kumar Nayak. "Enhanced modified LEACH (EMODLEACH) protocol for WSN." Advanced Computing and Communication (ISACC), 2015 International Symposium on. IEEE, 2015.

[5] Faisal, S., et al. "Z-SEP: Zonal-stable election protocol for wireless sensor networks." arXiv preprint arXiv:1303.5364 (2013).

[6] Lavita Virmani, S.K. Agarwal, Dharam Vir "Energy Efficient Cluster Based Multihop Routing for Wireless Sensor Network" International Journal of Advanced Research in Electrical, Electronics and Instrumentation Engineering, Vol. 3, Issue 10, October 2014. Pp. 12575 12586.

[7] Shweta Sharma, Tarun Kumar "Energy Efficient Modified Cluster Routing Algorithm for Maximizing Stability Period of WSN" International Journal of Innovative Research in Computer and Communication Engineering (An ISO 3297: 2007 Certified Organization) Vol. 3, Issue 9, September 2015. Pp. 8423-8428.

[8] Kirankumar B. Balavalad, Ajaykumar C. Katageri, Balaji M. Biradar, Deepa Chavan, and Basavaraj M. Angadi "Multipath-LEACH an Energy Efficient Routing Algorithm for Wireless Sensor Network" Journal of Advances in Computer Networks, Vol. 2, No. 3, September 2014.

[9] Taruna, S., Rekha Kumawat, and G. N. Purohit. "Multihop clustering protocol using gateway nodes in wireless sensor network." International Journal of Wireless \& Mobile Networks 4.4 (2012): 169 